\title{
Dark Matter Equation of State through Cosmic History
}

DOI:

10.1103/PhysRevLett.120.221102

\section{Document Version}

Final published version

Link to publication record in Manchester Research Explorer

\section{Citation for published version (APA):}

Kopp, M., Skordis, C., Thomas, D. B., \& Ili, S. (2018). Dark Matter Equation of State through Cosmic History. Physical Review Letters, 120(22). https://doi.org/10.1103/PhysRevLett.120.221102

\section{Published in:}

Physical Review Letters

\section{Citing this paper}

Please note that where the full-text provided on Manchester Research Explorer is the Author Accepted Manuscript or Proof version this may differ from the final Published version. If citing, it is advised that you check and use the publisher's definitive version.

\section{General rights}

Copyright and moral rights for the publications made accessible in the Research Explorer are retained by the authors and/or other copyright owners and it is a condition of accessing publications that users recognise and abide by the legal requirements associated with these rights.

\section{Takedown policy}

If you believe that this document breaches copyright please refer to the University of Manchester's Takedown Procedures [http://man.ac.uk/04Y6Bo] or contact uml.scholarlycommunications@manchester.ac.uk providing relevant details, so we can investigate your claim.

\section{OPEN ACCESS}




\title{
Dark Matter Equation of State through Cosmic History
}

\author{
Michael Kopp, ${ }^{1,2, *}$ Constantinos Skordis, ${ }^{1,2, \dagger}$ Daniel B Thomas, ${ }^{3,2,}$ and Stéphane Ilici ${ }^{1, \S}$ \\ ${ }^{1}$ CEICO, Institute of Physics of the Czech Academy of Sciences, Na Slovance 2, Praha 8, Czech Republic \\ ${ }^{2}$ Department of Physics, University of Cyprus, 1, Panepistimiou Street, 2109 Aglantzia, Cyprus \\ ${ }^{3}$ Jodrell Bank Centre for Astrophysics, School of Physics and Astronomy, The University of Manchester, \\ Manchester M13 9PL, United Kingdom
}

(Received 28 February 2018; revised manuscript received 30 April 2018; published 1 June 2018)

\begin{abstract}
Cold dark matter is a crucial constituent of the current concordance cosmological model. Having a vanishing equation of state (EOS), its energy density scales with the inverse cosmic volume and is thus uniquely described by a single number, its present abundance. We test the inverse cosmic volume law for dark matter (DM) by allowing its EOS to vary independently in eight redshift bins in the range $z=10^{5}$ and $z=0$. We use the latest measurements of the cosmic microwave background radiation from the Planck satellite and supplement them with baryon acoustic oscillation (BAO) data from the 6dF and SDSS-III BOSS surveys and with the Hubble Space Telescope (HST) key project data. We find no evidence for nonzero EOS in any of the eight redshift bins. With Planck data alone, the DM abundance is most strongly constrained around matter-radiation equality $\omega_{g}^{\mathrm{eq}}=0.1193_{-0.0035}^{+0.0036}$ (95\% C.L.), whereas its present-day value is more weakly constrained: $\omega_{g}^{(0)}=0.16_{-0.10}^{+0.12}$ (95\% C.L.). Adding BAO or HST data does not significantly change the $\omega_{g}^{\mathrm{eq}}$ constraint, while $\omega_{g}^{(0)}$ tightens to $0.160_{-0.065}^{+0.069}$ (95\% C.L.) and $0.124_{-0.067}^{+0.081}$ (95\% C.L.), respectively. Our results constrain for the first time the level of "coldness" required of the DM across various cosmological epochs and show that the DM abundance is strictly positive at all times.
\end{abstract}

DOI: 10.1103/PhysRevLett.120.221102

Introduction.-Cosmological observations indicate that there is insufficient baryonic matter in the Universe for the correct description of physical processes, if gravitational laws are dictated by general relativity. A natural explanation is that most of the matter fields interact negligibly with light, and are thus called dark matter, but can still be seen through their gravitational effect.

Dark matter (DM) is generally thought to be a stable particle (or particles) not part of the standard model; however, it has so far remained elusive [1-7]. Cosmologically, it is usually modeled as cold dark matter (CDM), which is part of the successful $\Lambda C D M$ model that is consistent with observations of the cosmic microwave background (CMB) (e.g., [8]), cosmic shear surveys (e.g., [9]), measurements of the background expansion such as BAO probes [10], supernovae distance measurements [11], and the observed abundance of light elements [12].

The CDM model is defined by a phase space distribution function satisfying the collisionless Boltzmann equation with an initially vanishing velocity dispersion and curl. This leads to a background CDM density $\bar{\rho}_{c}(a) \propto a^{-3}(a$ being the scale factor of the Universe) and equation of state (EOS) $w=0$, while the linearized density and velocity perturbations satisfy the continuity and pressureless Euler equations. Note that we use $w$ to indicate the EOS of DM and not the EOS of dark energy which we assume to be -1 as in $\Lambda$ CDM. The resulting model arises naturally in the weakly interacting massive particle paradigm: The candidate particles are effectively collisionless, typically have an EOS $w \sim 10^{-24} a^{-2}[13,14]$ and, thus, are well described by CDM. The QCD axion is another CDM candidate [15].

Not all DM candidates fit into the CDM paradigm, for instance, warm DM [14,16,17], ultralight axions [18,19], collisionless massive neutrinos [20,21], self-interacting massive neutrinos [22,23], Chaplygin gas [24], and selfinteracting DM [25]. In addition, DM may interact with other species such as neutrinos [26,27], photons [28,29], dark radiation [30-33], and dark energy [34-36].

Rather than taking the CDM description for granted, we consider it timely to examine whether the data themselves support any deviation from the CDM paradigm and, thus, to further determine or constrain DM properties. For our purpose, we use the generalized dark matter (GDM) model, first proposed by $\mathrm{W}$. Hu [37]. The phenomenology of the GDM model has been thoroughly investigated in Ref. [38], where a connection was found with more fundamental theories, including those of a rich self-interacting dark sector. In addition, the recent work on the effective field theory of large-scale structure (EFT of LSS) [39] suggests that, even for an initially pressureless perfect fluid, the nonlinearities that develop on small scales affect the cosmological background and large-scale linear perturbations, creating an effective pressure and viscosity such as those found in GDM. 
The GDM model has been used to constrain DM properties with either constant or specific time dependences of the parameters [40-45]. Here, we allow the DM EOS to vary more freely in time than all previous studies.

The model.-We consider a flat Universe with only scalar perturbations; see [38] for more details and notation. The background density $\bar{\rho}_{g}$ and pressure $\bar{P}_{g}$ of the DM evolve according to the conservation law

$$
\dot{\bar{\rho}}_{g}=-3 H(1+w) \bar{\rho}_{g}, \quad \bar{P}_{g}=w \bar{\rho}_{g}, q
$$

where $H=(\dot{a} / a)$ is the Hubble parameter and the overdot denotes derivatives with respect to cosmic time $t$. The parametric function $w(t)$ is freely specifiable with the case $w=0$ corresponding to a CDM background $\left(\bar{\rho}_{g}=\bar{\rho}_{c}\right)$. The GDM model has two further free functions: the speed of sound $c_{s}^{2}$ and the (shear) viscosity $c_{\text {vis. The }}^{2} \operatorname{EOS} w$ is uncorrelated with the two perturbative parameters $c_{s}^{2}$ and $c_{\mathrm{vis}}^{2}$, as shown in Ref. [44]; thus, in this work, we set these two to zero and denote this class of GDM models by $w \mathrm{DM}$. Consequently, replacing CDM by $w \mathrm{DM}$ in the $\Lambda \mathrm{CDM}$ model leads to $\Lambda w \mathrm{DM}$.

With this choice, the perturbed $w \mathrm{DM}$ fluid equations for the density contrast $\delta_{g}$ and velocity perturbation $\theta_{g}$ are given by

$$
\begin{aligned}
\frac{\dot{\delta}_{g}}{1+w} & =3 H\left(\frac{w \delta_{g}}{1+w}+3 a H c_{a}^{2} \theta_{g}\right)-\left(\frac{1}{2} \dot{h}-\frac{1}{a} \vec{\nabla}^{2} \theta_{g}\right), \\
\dot{\theta}_{g} & =-H \theta_{g}, \quad c_{a}^{2}=\frac{\dot{\bar{P}}_{g}}{\dot{\bar{\rho}}_{g}}=w-\frac{\dot{w}}{3 H(1+w)} .
\end{aligned}
$$

Here, $c_{a}^{2}$ is the adiabatic speed of sound and $h$ is a metric perturbation in the synchronous gauge $[37,38]$. The Euler equation $\dot{\theta}_{g}=-H \theta_{g}$ is identical to that of CDM, which implies the solution $\theta_{g}=0$. An example of $w \mathrm{DM}$ is the combination of CDM and $\Lambda$ interpreted as a single fluid with $w=-\left(1+\bar{\rho}_{c} / \bar{\rho}_{\Lambda}\right)^{-1}$. A large degeneracy between $\Omega_{\Lambda}$ and $w$ is thus expected at late times (see also [46]).

Methodology.-The $w \mathrm{DM}$ fluid equations (1) and (2) were implemented in the Boltzmann code CLASS [47] as in Refs. $[38,44]$. A sufficiently general time dependence of $w$ was achieved by binning its evolution into $N=8$ scale factor bins, whose edges are $\tilde{a}_{i}=$ $10^{\{0,-1,-1.5,-2,-2.5,-3,-3.5,-4\}}$. The bins were smoothly connected using $w(a)=\operatorname{erf}\left[\sigma_{a}^{-1} \ln \left(a / \tilde{a}_{i+1}\right)\right]\left(w_{i}-w_{i+1}\right) / 2+$ $\left(w_{i}+w_{i+1}\right) / 2$ for $a_{i+1}<a<a_{i}$, with bin centers $a_{i}=$ $\sqrt{\tilde{a}_{i} \tilde{a}_{i+1}}$ for $1 \leq i \leq N-2$ while $a_{0}=1$ and $a_{N-1}=0$. Because of the aforementioned degeneracy of $w \mathrm{DM}$ with $\mathrm{CDM}$ and $\Lambda$, we chose a wider bin in the late Universe.

The $\sigma_{a}$ parameter controls the transition width between bins; it was set to $1 / 20$ so that the transition is small compared to the bin width. We tested that this choice does not affect our conclusions.

We define a dimensionless scaled $w \mathrm{DM}$ density

$$
\omega_{g} \equiv a^{3} \bar{\rho}_{g} \frac{8 \pi G}{3 \times(100 \mathrm{~km} / \mathrm{s} / \mathrm{Mpc})^{2}} .
$$

When $w=0$ through cosmic history, $\omega_{g}$ is a constant equal to the conventional dimensionless CDM density $\omega_{c}$. In general, however, $\omega_{g}$ varies over time and is fully determined by the $N+1$ parameters $\omega_{g}^{(0)}$ and $w_{i}$. We use the notation $\omega_{g}^{(i)}=\omega_{g}\left(a_{i}\right)$ and similarly for other functions with subscripts, so that the present-day DM abundance is $\omega_{g}^{(0)}=\omega_{g}\left(a_{0}\right)$. For functions without a subscript, we instead write $H_{i}=H\left(a_{i}\right)$ and $w_{i}=w\left(a_{i}\right)$.

Our parameter constraints were obtained as in Ref. [44], and we present only brief details here. We used the Markov chain Monte Carlo code MontePyThon [48] and established a convergence of the chains using the Gelman-Rubin criterion [49]. Our total parameter set

$$
\left(\omega_{b}, \omega_{g}^{(0)}, H_{0}, n_{s}, \tau, \ln 10^{10} A_{s}, w_{i}\right)
$$

consists of six $\Lambda \mathrm{CDM}$ parameters and the eight values $w_{i}$. We denote the $\Lambda w$ DM model with eight bins as "var- $w$ " and the previously studied model [44] with $w=$ const as "const- $w$." We assumed adiabatic initial conditions.

We used the Planck 2015 data release [50] of the CMB anisotropies power spectra, composed of the low- $l$ T/E/B likelihood and the full TT/TE/EE high- $l$ likelihood with the complete "not-lite" set of nuisance parameters [51]. These likelihoods combined are referred to as Planck power spectra (PPS). We also added selectively the HST key project prior on $H_{0}$ [52], BAO from the 6dF Galaxy Survey [53] and the Baryon Oscillation Spectroscopic Survey Sloan Digital Sky Survey [10], and the Planck CMB lensing likelihood (respectively referred to as HST, $\mathrm{BAO}$, and lens hereafter).

We set uniform priors on $\tau$ and $H_{0}$ such that $0.01<\tau$ and $45 \leq H_{0} \leq 90$, respectively. We used the same priors on Planck nuisance parameters and the same neutrino treatment as in Ref. [44]. The helium fraction was set to $Y_{\mathrm{He}}=0.24667$ [8].

Results.-Our main results are constraints on the time dependence of DM EOS $w(a)$ and abundance $\omega_{g}(a)$ shown in Figs. 1 and 2. For comparison, we also show the constraints on the const- $w$ model already discussed in Ref. [44]. We list the $95 \%$ confidence regions of all parameters in Table I.

In Fig. 1, we observe that $\Lambda \mathrm{CDM}$ lies in the 99\% confidence region of the const- $w$ model, which in turn lies in the $99 \%$ confidence region of the var- $w$ model, such that the constraints are nested like the models themselves. There is no evidence for significant deviations of the DM EOS from 


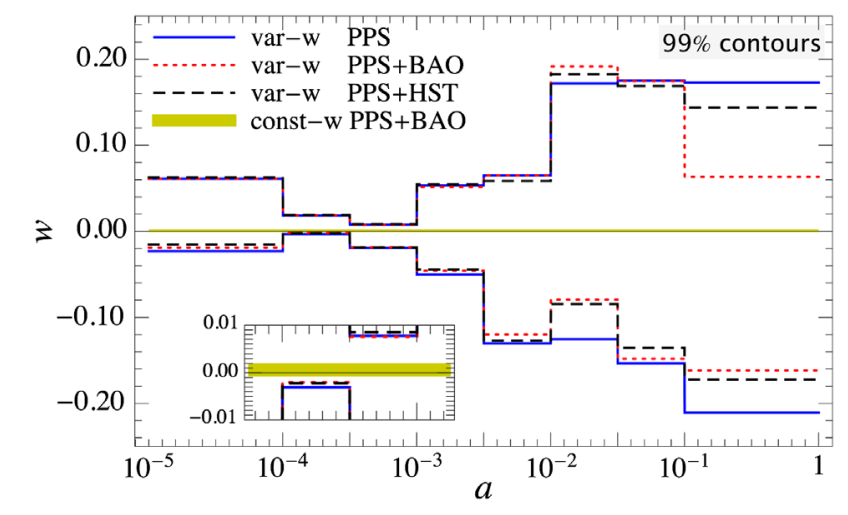

FIG. 1. The $99 \%$ confidence regions on the EOS of DM, $w(a)$ for $\sigma_{a}=0$. The eight bins are indicated by the large ticks on the $a$ axis. The different line styles correspond to different data sets and models specified in the legend. The inset shows the region between $\tilde{a}_{7}=10^{-4}$ and $\tilde{a}_{5}=10^{-3}$ magnified. Within the const$w$ narrow stripe lies the $\Lambda$ CDM model indicated by the black solid line.

0 at any time. Consequently, any model selection criteria will favor $\Lambda \mathrm{CDM}$.

The constraints on $w$ are the strongest between $a_{6}$ and $a_{5}$ enclosing the matter-radiation equality $a_{\mathrm{eq}} \simeq 3 \times 10^{-4}$ and are about a factor of 2 weaker compared to the const- $w$ model. In other bins, the constraints on $w$ weaken significantly. Adding the BAO or HST data set has only a minor effect on var- $w$ constraints and only tightens limits in the rightmost bin. As was the case for the const- $w$ model [44], adding $\mathrm{CMB}$ lensing does not significantly improve the constraints.

Let us now compare in more detail the DM abundance $\omega_{g}(a)$ of the var- $w$ and const $-w$ models focusing only on the two data set combinations PPS and PPS + BAO + lens. In Fig. 2, we see that, like $w(a), \omega_{g}$ is most tightly constrained between $a_{6}=10^{-3.75}$ and $a_{5}=10^{-3.25}$, in fact almost as

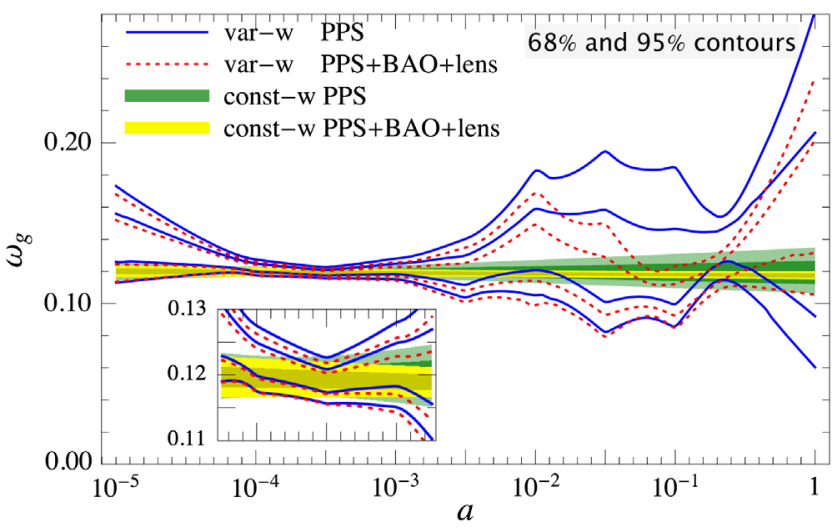

FIG. 2. The $68 \%$ and $95 \%$ contours of the $1 \mathrm{D}$ marginalized posteriors on the DM abundance $\omega_{g}(a)$. We show the var- $w$ and const- $w$ models, both with two different data sets (PPS and $\mathrm{PPS}+\mathrm{BAO}+$ lens) as specified in the legend. tightly as for the const- $w$ model (see the inset in Fig. 2). Around $a=0.4$, there is a squeeze in the constraints of $\omega_{g}$ from PPS, which extends to $a \sim(0.08,0.4)$ when BAO or HST are included. At all times, a vanishing DM abundance $\left(\omega_{g}=0\right)$ is inconsistent with the data. More quantitatively, we find for the var- $w$ model at 95\% C.L. $\omega_{g}^{\mathrm{eq}}=$ $0.1193_{-0.0035}^{+0.0036}$ and $\omega_{g}^{(0)}=0.16_{-0.10}^{+0.12}$ with PPS only, whereas for PPS $+\mathrm{BAO}+$ lens we get $\omega_{g}^{\mathrm{eq}}=0.1189_{-0.0033}^{+0.0032}$ and $\omega_{g}^{(0)}=0.169_{-0.065}^{+0.067}$. For const- $w$-PPS $+\mathrm{BAO}+$ lens, we find $\omega_{g}^{\mathrm{eq}}=0.1193_{-0.0026}^{+0.0026}$, whereas the $\Lambda \mathrm{CDM}$ result is $\omega_{c}=\omega_{g}^{\mathrm{eq}}=0.1184_{-0.0022}^{+0.0022}$; see [44].

Consider the tightly constrained region around $a_{\mathrm{eq}}$, $a_{6}<a<a_{5}$, as shown in Fig. 2 (see also the inset in the same figure). As discussed in Refs. [37,38], the GDM abundance $\omega_{g}(a)$ and expansion rate $H(a)$ in the early Universe determine the time of matter-radiation equality and thereby the amount of potential decay until recombination. This in turn sets the relative heights of the first few peaks of the CMB temperature angular power spectrum. Both the const $w$ and var- $w$ models constrain $\omega_{g}$ around $a_{\mathrm{eq}}$ at a similar level (see above). The degeneracy between $H_{0}$ and $\omega_{g}$ in the const- $w$ model [44] translates into a degeneracy between $H_{6}$ and $\omega_{g}^{(6)}$ in the var- $w$ model as seen in the left panel in Fig. 3. Indeed, the $H_{6}-\omega_{g}^{(6)}$ contours reveal how well the CMB constrains a combination of the expansion rate and the abundance of DM around $a_{\mathrm{eq}}$. The

TABLE I. The $95 \%$ confidence intervals of var- $w$ parameters.

\begin{tabular}{lccc}
\hline \hline \multicolumn{3}{c}{ Data } \\
\hline Parameter & PPS & PPS + BAO & PPS + HST \\
\hline $100 \omega_{b}$ & $2.221_{-0.040}^{+0.041}$ & $2.217_{-0.038}^{+0.040}$ & $2.218_{-0.038}^{+0.039}$ \\
$\omega_{g}^{(0)}$ & $0.16_{-0.10}^{+0.12}$ & $0.160_{-0.065}^{+0.069}$ & $0.124_{-0.067}^{+0.081}$ \\
$H_{0}[\mathrm{~km} / \mathrm{s} / \mathrm{Mpc}]$ & $<65.9$ & $66.6_{-4.0}^{+3.7}$ & $72.3_{-4.6}^{+4.5}$ \\
$n_{s}$ & $0.974_{-0.020}^{+0.021}$ & $0.974_{-0.020}^{+0.020}$ & $0.977_{-0.020}^{+0.020}$ \\
$\tau$ & $0.072_{-0.035}^{+0.039}$ & $0.076_{-0.034}^{+0.040}$ & $0.076_{-0.034}^{+0.037}$ \\
$\ln \left(10^{10} A_{s}\right)$ & $3.085_{-0.069}^{+0.076}$ & $3.096_{-0.067}^{+0.078}$ & $3.096_{-0.067}^{+0.073}$ \\
$w_{0}$ & $-0.03_{-0.14}^{+0.17}$ & $-0.056_{-0.083}^{+0.091}$ & $-0.01_{-0.13}^{+0.12}$ \\
$w_{1}$ & $0.01_{-0.13}^{+0.13}$ & $0.02_{-0.13}^{+0.12}$ & $0.02_{-0.12}^{+0.12}$ \\
$w_{2}$ & $0.02_{-0.11}^{+0.12}$ & $0.05_{-0.10}^{+0.10}$ & $0.05_{-0.10}^{+0.11}$ \\
$w_{3}$ & $-0.044_{-0.072}^{+0.075}$ & $-0.036_{-0.068}^{+0.072}$ & $-0.041_{-0.067}^{+0.075}$ \\
$w_{4}$ & $0.002_{-0.039}^{+0.038}$ & $0.005_{-0.038}^{+0.036}$ & $0.005_{-0.038}^{+0.038}$ \\
$w_{5}$ & $-0.006_{-0.010}^{+0.011}$ & $-0.006_{-0.010}^{+0.010}$ & $-0.005_{-0.010}^{+0.011}$ \\
$w_{6}$ & $0.0078_{-0.0081}^{+0.0079}$ & $0.0084_{-0.0079}^{+0.0078}$ & $0.0085_{-0.0080}^{+0.0080}$ \\
$w_{7}$ & $0.021_{-0.032}^{+0.031}$ & $0.022_{-0.031}^{+0.030}$ & $0.025_{-0.030}^{+0.029}$ \\
$\Omega_{\Lambda}^{(0)}$ & $0.34_{-0.58}^{+0.45}$ & $0.58_{-0.21}^{+0.18}$ & $0.72_{-0.16}^{+0.14}$ \\
$\sigma_{8}$ & $0.71_{-0.36}^{+0.45}$ & $0.72_{-0.23}^{+0.27}$ & $0.91_{-0.39}^{+0.43}$ \\
\hline \hline
\end{tabular}




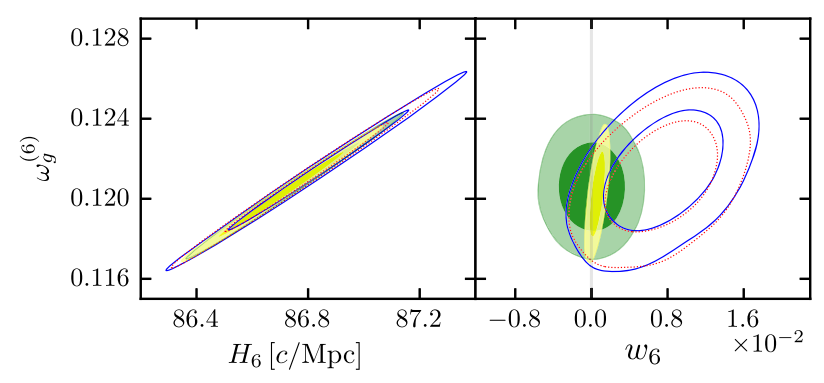

FIG. 3. The $68 \%$ and $95 \%$ contours of 2D marginalized posteriors of $\omega_{g}^{(6)}-H_{6}$ (left) and $\omega_{g}^{(6)}-w_{6}$ (right). The line styles and colors are as in Figs. 2 and 4.

degeneracy between $w-\omega_{g}[38,44]$ in the const- $w$ model due to the same effect is also seen as a degeneracy between $w_{6}$ and $\omega_{g}^{(6)}$ (right panel in Fig. 3); however, in the var- $w$ model, it is weakened, as $w_{6}$ has only an indirect effect on $a_{\text {eq }}$, contrary to $\omega_{g}^{(6)}$. Similar correlations exist for $H_{5}-\omega_{g}^{(5)}$ and $\omega_{g}^{(5)}-w_{5}$ but in the opposite direction.

The origin of the squeeze around $a \sim(0.08-0.4)$ is of an entirely different nature. The angular diameter distance $d_{A}^{*}$ to the last scattering surface is given by

$$
d_{A}^{*}=a_{*} \int_{a_{*}}^{1} d \ln a(a H)^{-1}=a_{*}\left(\eta_{0}-\eta_{*}\right) .
$$

Here, the second equality has been written in terms of the conformal time $\left(\eta=\int d t / a\right)$ today, $\eta_{0}$, and at the last scattering surface, $\eta_{*}$. The largest contribution to $d_{A}^{*}$ comes from the first $\ln a$ bin where $\eta$ grows from $\sim 4000$ to $\sim 14000 \mathrm{Mpc}$ and constitutes $\sim 70 \%$ of the total. This contribution can be strongly constrained by geometric probes. Within the first scale factor bin, we have $H=$ $\sqrt{\omega_{g}^{(0)}\left(a^{-3\left(1+w_{0}\right)}-1\right)+H_{0}^{2}}$ (with $H$ and $H_{0}$ in units of $100 \mathrm{~km} / \mathrm{s} / \mathrm{Mpc}$ ). Hence, for a one-parameter family of $\omega_{g}^{(0)}$ and $w_{0}$, the combination $(a H)^{-1}$ is approximately constant. As this is the largest contributor to $d_{A}^{*}$, we expect $w_{0}$ and $\omega_{g}^{(0)}$ to be anticorrelated, as is indeed observed in Fig. 4 (lower left panel). The inclusion of BAO or HST data significantly improves and shifts the constraints on $\eta(a)$ and is, in turn, reflected in the $\omega_{g}$ and $w$ constraints.

For the var- $w$ model, PPS alone allows for very low $H_{0}$, as low as $45 \mathrm{~km} / \mathrm{s} / \mathrm{Mpc}$, corresponding to our hard prior on $H_{0}$; see the blue contours in the top left and bottom right panels in Fig. 4. Adding BAO (red dotted lines) or HST (black dashed lines) shrinks the posteriors and also moves the mean of $H_{0}$ back towards the range consistent with the const- $w$ model [44]. BAO (and also HST) data lead to a degeneracy between $H_{0}$ and $w_{0}$ and between $H_{0}$ and $\omega_{g}^{(0)}$, as in the const- $w$ model. However, as the present-day values of $H$ and $\omega_{g}$ are no longer anchored to their early Universe

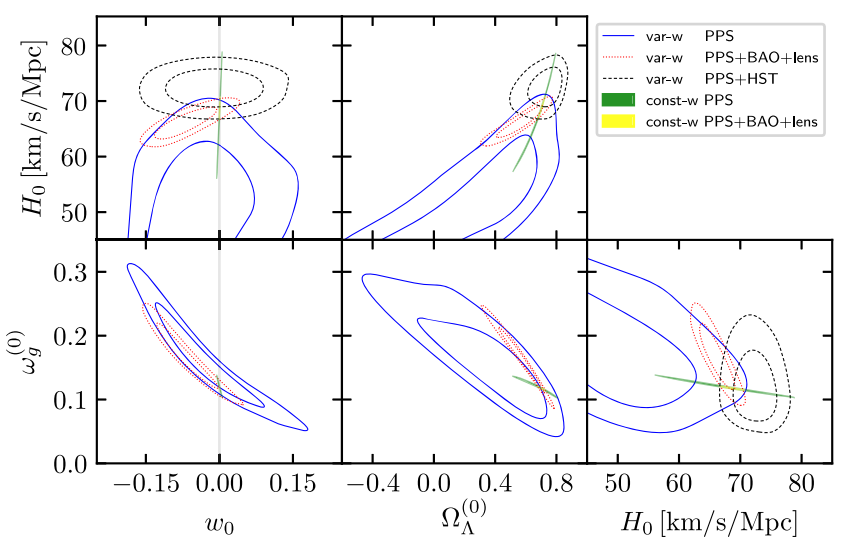

FIG. 4. The $68 \%$ and $95 \%$ contours of $2 \mathrm{D}$ marginalized posteriors for combinations of parameters in the set $\left\{H_{0}, w_{0}, \omega_{g}^{(0)}, \Omega_{\Lambda}\right\}$. The PPS + HST contours in the $\omega_{g}^{(0)}-w_{0}$ and $\omega_{g}^{(0)}-\Omega_{\Lambda}^{(0)}$ panels are not displayed, as they are very similar to $\mathrm{PPS}+\mathrm{BAO}(+$ lens $)$.

values, the degeneracy axis is rotated and the contours are not as flattened.

In the middle panels in Fig. 4, we display the 2D marginalized posteriors of the $H_{0}-\Omega_{\Lambda}$ and $\omega_{g}^{(0)}-\Omega_{\Lambda}$ planes. As $\omega_{b}$ is well constrained, $w \mathrm{DM}$ and $\Lambda$ are the only relevant species in the late (flat) Universe and are expected to have their abundances anticorrelated. Indeed, the parameter $\omega_{g}^{(0)}$ is anticorrelated with $\Omega_{\Lambda}^{(0)}$ for all data sets. The combination of CDM and $\Lambda$ may be modeled by $w \mathrm{DM}$; in that model, however, $w$ changes steeply only within the $w_{0}$ bin so that this behavior is unaffected. When BAO or HST data are included, the slope and size of the contours change strongly as the late Universe behavior dissociates from the early Universe in the var- $w$ model. The negative values of $\Omega_{\Lambda}^{(0)}$ are correlated with the low values of $H_{0}$, and, while allowed by PPS, they disappear when $H_{0}$ is better constrained after including BAO or HST data.

Implications.-In the $w \mathrm{DM}$ model, the DM abundance $\omega_{g}$ may deviate from its expected (constant) CDM value throughout cosmic history, causing only minimal changes to the clustering properties of DM. Hence, the constraints on $w$ and $\omega_{g}$ are conservative. One could also conservatively allow for general $c_{s}^{2}(a, k)$ and $c_{\mathrm{vis}}^{2}(a, k)$ and marginalize over them. However, as $w$ is almost uncorrelated with $c_{s}^{2}$ and $c_{\text {vis }}^{2}$, we expect such a procedure to give constraints similar to those here. In the cases of warm DM and EFT of LSS, the parameters $w, c_{s}^{2}$, and $c_{\mathrm{vis}}^{2}$ are interrelated so that the $w$ constraints will be driven by $c_{s}^{2}$ and $c_{\text {vis }}^{2}$ and, hence, tightened further [14,45]. Adding a spatial curvature and/or neutrino mass would likely widen the $\omega_{g}$ constraints on the squeeze at $a \sim\{0.08-0.4\}$ [54] and in the latter case on the tightly constrained region around $a_{\mathrm{eq}}$ as well. 
When applying our constraints to generic theories of dark matter, including those coming from modifications of gravity, one must keep in mind our underlying assumption of adiabaticity. As models of modified gravity will typically have additional fields leading to more types of isocurvature modes, we expect our constraints to be less applicable in those cases. However, within our adiabatic assumption, we expect our constraints to be valid for any theory of dark matter or modified gravity. The cosmological background in any such theory will have to evolve as in $\Lambda \mathrm{CDM}$ (see, for example, [55]), around matter-radiation equality, and before decoupling. Typical examples include DM-DE coupled models [34-36]. Explicit realizations where a CDM-like background decays into DE are given by the quasidilaton models of massive gravity [56,57] and by axion models [58].

Conclusion.-We have constrained the EOS $w$ and abundance $\omega_{g}$ of dark matter, in eight temporal bins covering five decades in cosmic scale factor, using the CMB data from the Planck satellite, and separately including BAO and HST data. We found that $w$ is consistent with zero and the DM abundance is strictly positive at all cosmological epochs considered here (see Figs. 1 and 2), and thus the concordance $\Lambda \mathrm{CDM}$ model remains unchallenged. This is the first time that the level of DM "coldness" across cosmic time has been explicitly constrained.

The research leading to these results has received funding from the European Research Council under the European Union's Seventh Framework Program (FP7/ 2007-2013)/ERC Grant Agreement No. 617656 "Theories and Models of the Dark Sector: Dark Matter, Dark Energy and Gravity." The primary investigator is C. S.

*kopp@fzu.cz

†skordis@fzu.cz

"daniel.thomas-2@manchester.ac.uk

\$ilic@fzu.cz

[1] R. Agnese et al., Phys. Rev. Lett. 116, 071301 (2016).

[2] A. Tan et al., Phys. Rev. Lett. 117, 121303 (2016).

[3] E. Aprile et al., Phys. Rev. D 94, 092001 (2016).

[4] J. V. Sloan et al., Phys. Dark Universe 14, 95 (2016).

[5] D. S. Akerib et al., Phys. Rev. Lett. 118, 021303 (2017).

[6] F. Petricca et al. (CRESST Collaboration), arXiv:1711 .07692 .

[7] B. M. Brubaker et al., Phys. Rev. Lett. 118, 061302 (2017).

[8] P. A. R. Ade et al. (Planck Collaboration), Astron. Astrophys. 594, A13 (2016).

[9] T. M. C. Abbott et al., arXiv:1801.03181.

[10] L. Anderson et al., Mon. Not. R. Astron. Soc. 441, 24 (2014).

[11] D. O. Jones et al., Astrophys. J. 857, 51 (2018).

[12] M. Peimbert, V. Luridiana, and A. Peimbert, Astrophys. J. 666, 636 (2007).

[13] A. M. Green, S. Hofmann, and D. J. Schwarz, J. Cosmol. Astropart. Phys. 08 (2005) 003.
[14] C. Armendariz-Picon and J. T. Neelakanta, J. Cosmol. Astropart. Phys. 03 (2014) 049.

[15] L. Visinelli and P. Gondolo, Phys. Rev. Lett. 113, 011802 (2014).

[16] S. Dodelson and L. M. Widrow, Phys. Rev. Lett. 72, 17 (1994).

[17] O. F. Piattella, L. Casarini, J. C. Fabris, and J. A. de Freitas Pacheco, J. Cosmol. Astropart. Phys. 02 (2016) 024.

[18] W. Hu, R. Barkana, and A. Gruzinov, Phys. Rev. Lett. 85, 1158 (2000).

[19] R. Hlozek, D. Grin, D. J. E. Marsh, and P. G. Ferreira, Phys. Rev. D 91, 103512 (2015).

[20] M. Shoji and E. Komatsu, Phys. Rev. D 82, 089901(E) (2010).

[21] J. Lesgourgues and T. Tram, J. Cosmol. Astropart. Phys. 09 (2011) 032.

[22] F.-Y. Cyr-Racine and K. Sigurdson, Phys. Rev. D 90, 123533 (2014).

[23] I. M. Oldengott, C. Rampf, and Y. Y. Y. Wong, J. Cosmol. Astropart. Phys. 04 (2015) 016.

[24] H. B. Sandvik, M. Tegmark, M. Zaldarriaga, and I. Waga, Phys. Rev. D 69, 123524 (2004).

[25] D. N. Spergel and P. J. Steinhardt, Phys. Rev. Lett. 84, 3760 (2000).

[26] P. Serra, F. Zalamea, A. Cooray, G. Mangano, and A. Melchiorri, Phys. Rev. D 81, 043507 (2010).

[27] R. J. Wilkinson, C. Boehm, and J. Lesgourgues, J. Cosmol. Astropart. Phys. 05 (2014) 011.

[28] C. Boehm, A. Riazuelo, S. H. Hansen, and R. Schaeffer, Phys. Rev. D 66, 083505 (2002).

[29] R. J. Wilkinson, J. Lesgourgues, and C. Boehm, J. Cosmol. Astropart. Phys. 04 (2014) 026.

[30] F.-Y. Cyr-Racine and K. Sigurdson, Phys. Rev. D 87, 103515 (2013).

[31] R. Diamanti, E. Giusarma, O. Mena, M. Archidiacono, and A. Melchiorri, Phys. Rev. D 87, 063509 (2013).

[32] M. A. Buen-Abad, G. Marques-Tavares, and M. Schmaltz, Phys. Rev. D 92, 023531 (2015).

[33] J. Lesgourgues, G. Marques-Tavares, and M. Schmaltz, J. Cosmol. Astropart. Phys. 02 (2016) 037.

[34] L. Amendola, Mon. Not. R. Astron. Soc. 312, 521 (2000).

[35] A. Pourtsidou, C. Skordis, and E. J. Copeland, Phys. Rev. D 88, 083505 (2013).

[36] G. D'Amico, T. Hamill, and N. Kaloper, Phys. Rev. D 94, 103526 (2016).

[37] W. Hu, Astrophys. J. 506, 485 (1998).

[38] M. Kopp, C. Skordis, and D. B. Thomas, Phys. Rev. D 94, 043512 (2016).

[39] D. Baumann, A. Nicolis, L. Senatore, and M. Zaldarriaga, J. Cosmol. Astropart. Phys. 07 (2012) 051.

[40] C. M. Müller, Phys. Rev. D 71, 047302 (2005).

[41] E. Calabrese, M. Migliaccio, L. Pagano, G. DeTroia, A. Melchiorri, and P. Natoli, Phys. Rev. D 80, 063539 (2009).

[42] S. Kumar and L. Xu, Phys. Lett. B 737, 244 (2014).

[43] L. Xu and Y. Chang, Phys. Rev. D 88, 127301 (2013).

[44] D. B. Thomas, M. Kopp, and C. Skordis, Astrophys. J. 830, 155 (2016).

[45] M. Kunz, S. Nesseris, and I. Sawicki, Phys. Rev. D 94, 023510 (2016). 
[46] I. Tutusaus, B. Lamine, A. Blanchard, A. Dupays, Y. Rousset, and Y. Zolnierowski, Phys. Rev. D 94, 123515 (2016).

[47] D. Blas, J. Lesgourgues, and T. Tram, J. Cosmol. Astropart. Phys. 07 (2011) 034.

[48] B. Audren, J. Lesgourgues, K. Benabed, and S. Prunet, J. Cosmol. Astropart. Phys. 02 (2013) 001.

[49] D. B. R. Andrew Gelman, Stat. Sci. 7, 457 (1992).

[50] N. Aghanim et al. (Planck Collaboration), Astron. Astrophys. 594, A11 (2016).

[51] For full details, see the Planck papers and wiki http://wiki .cosmos.esa.int/planckpla2015/index.php/.

[52] A. G. Riess, L. Macri, S. Casertano, H. Lampeitl, H. C. Ferguson, A. V. Filippenko, S. W. Jha, W. Li, and R. Chornock, Astrophys. J. 730, 119 (2011).
[53] F. Beutler, C. Blake, M. Colless, D. H. Jones, L. Staveley-Smith, L. Campbell, Q. Parker, W. Saunders, and F. Watson, Mon. Not. R. Astron. Soc. 416, 3017 (2011).

[54] P. G. Ferreira, C. Skordis, and C. Zunckel, Phys. Rev. D 78, 044043 (2008).

[55] M. Banados, P. G. Ferreira, and C. Skordis, Phys. Rev. D 79, 063511 (2009).

[56] R. Gannouji, Md. Wali Hossain, M. Sami, and E. N. Saridakis, Phys. Rev. D 87, 123536 (2013).

[57] S. Anselmi, D. López Nacir, and G. D. Starkman, Phys. Rev. D 92, 084033 (2015).

[58] T. Kobayashi and P. G. Ferreira, arXiv:1801.09658. 